\title{
Endogenously Asymmetric Demand Shocks in a Monetary Union
}

\author{
Olivier Loisel \\ CREST-LMA, Paris, France
}

\begin{abstract}
This paper presents a two-country two-industry monetary model, with intermediate inputs and transport costs, which builds a bridge between the New Open Economy Macroeconomics and the New Economic Geography literatures. Endogenously asymmetric shocks arise in this model when the exchange rate regime in force fosters the concentration of each industry in one country, thus turning industry-specific shocks into country-specific shocks. Because of the conjunction of substitution and/or income effects, endogenously asymmetric demand shocks are found more likely to arise in a monetary union than under a flexible exchange rate regime.
\end{abstract}

- JEL Classifications: F12, F15, F33, F41, R12, R13

- Key words: Asymmetric shocks, Endogenous specialization,

Optimum currency area

\section{Introduction}

This paper investigates the possibility of endogenously asymmetric shocks under alternative exchange rate regimes, focusing on demand shocks. Endogenously asymmetric shocks arise when the exchange rate regime in force promotes greater national specialization, thus turning industry-specific shocks into country-specific shocks. The concept of endogenously asymmetric shocks goes back to the early 1990s, when the New Economic Geography theory emerged to show that a fall in

\footnotetext{
*Corresponding address: CREST-LMA, 15 Boulevard Gabriel Péri, 92245 Malakoff Cédex, France, Tel: +33-1-41-17-60-38, Fax: +33-1 41-17-76-66, E-mail: olivier.loisel@ensae.fr

(C)2005-Center for International Economics, Sejong Institution, All Rights Reserved.
} 
the cost of transport of goods and services across countries could affect the specialization patterns of these countries, for instance lead to more national specialization by providing an incentive to reap scale economies and agglomeration benefits. The European monetary union (EMU) could then have the same effect if the launch of the euro, as a further step on the road to economic integration, can be interpreted at first sight as a fall in the transport cost across member countries, through the complete elimination of exchange rate risk. In other words, the Optimal Currency Area (OCA) criterion identified by Kenen (1969), i.e. the degree of national economic diversification in the presence of industry-specific shocks, should be considered as endogenous. Pointing to the fact that the United States experience a higher degree of local specialization than Europe, Krugman (1993) thus argues that EMU will tend to develop inter-industry rather than intraindustry international trade, and hence favour the emergence of endogenously asymmetric shocks.

On the theoretical ground, very little has been done to modelize explicitly endogenously asymmetric shocks in a monetary union. Such a modelization would be welcome however, if only because the relevance of the argument exposed above, which consists in interpreting the elimination of exchange rate risk as a fall in the transport cost across countries, implicitly rests on two discutable assumptions: exchange rate variations are exogenous, and firms behave as risk-adverse agents. This naturally raises the question of what would happen, were these two assumptions to be relaxed. One may wish to relax the assumption of risk-adverse firms by sheer curiosity, as it seems no more relevant than the alternative assumption of risk-neutral firms. And one may wish to relax the assumption of exogenous exchange rate variations simply because it stands at odds with the core assumption of the OCA literature, namely the assumption that country-specific shocks are smoothed by endogenous exchange rate variations under a flexible exchange rate regime ${ }^{1}$. This paper aims at filling in this gap in the literature, as it presents a model of endogenously asymmetric shocks where exchange rate variations are endogenous and firms behave as risk-neutral agents.

To our knowledge, Ricci's $(1997,1998)$ models are so far the only ones to tackle

\footnotetext{
${ }^{1}$ If exchange rate variations are exogenous, then Krugman's point may apply, making country-specific shocks bigger or more frequent under a fixed exchange rate regime than under a flexible exchange rate regime. But then a flexible exchange rate regime may not be preferable to a fixed exchange rate regime, because it does not smooth country-specific shocks anyway: on the contrary, it brings additional noise into the system through these exogenous exchange rate variations.
} 
the issue of endogenously asymmetric shocks under fixed and flexible exchange rate regimes. These models do not belong stricto sensu to the New Economic Geography literature, as they do not modelize transport costs and hence do not feature forward and backward linkages. Nonetheless, they show explicitly that countries' specialization patterns depend on the exchange rate regime when firms behave as risk-adverse agents. In its main specification, Ricci's (1997) model is a one-period, two-country, two-industry monetary model, where the exchange rate (when flexible) adjusts endogenously to shocks so as to balance trade. The point is the following: under a flexible exchange rate regime, firms of a given industry have an incentive to locate in the country relatively specialized in this industry, because industry-specific shocks are smoothed there by endogenous exchange rate variations. Countries tend therefore to be more specialized under flexible than under fixed exchange rate regimes. Ricci's (1998) model is a one-period, n-country monetary model, where the exchange rate (when flexible) is by contrast an exogenous random variable. The point is the following: under a flexible exchange rate regime, firms have an incentive to locate in the same country so as to enjoy a large market with no exchange rate risk. Flexible exchange rate regimes tend therefore to promote the agglomeration of economic activity.

Like Ricci's (1997), our model is a two-country, two-industry, one-period monetary model, where the exchange rate (when flexible) adjusts endogenously to shocks so as to balance trade. But firms behave as risk-neutral agents in our model. Moreover, our model builds a bridge between the New Economic Geography and the New Open Economy Macroeconomics literatures. More precisely, we borrow the industrial clustering model of Fujita, Krugman and Venables (1999, chapter 16), which features intermediate inputs and transport costs, and we reformulate this model in a stochastic environment where exchange rate variations have real effects in the presence of short-run nominal wage rigidity. The traditional dispersion force (based on the local competition effect) and concentration forces (based on backward and forward linkages), familiar to the New Economic Geography literature, are present in our model. But new forces enter the stage when industryspecific demand shocks are considered. These new forces explain why, despite firms behaving as risk-neutral agents, countries' specialization patterns still depend on the exchange rate regime. The argument goes as follows.

Consider first the case where the two countries form a monetary union. In that case, asymmetric shocks are not smoothed. Let us focus on a firm of a given industry located in the country relatively specialized in the other industry. In case 
of an industry-specific demand shock, this firm is faced with two opposite effects, a substitution effect (as demand shifts from one industry to the other) and an income effect (as the firm's country gets poorer or wealthier, and this matters in the presence of transport costs). The conjunction of these two opposite effects turns this firm's production into a concave function of the stochastic parameter representing the industry-specific shock. To have the same average production as firms of the other industry in the same country, this firm needs therefore to have lower prices and hence lower wages, so that households prefer to work for the firms of the other industry: a new concentration force arises.

Now consider the case of a flexible exchange rate regime. Asymmetric shocks are then smoothed by endogenous exchange rate variations, so that the income effect mentioned above disappears, and so does the newly identified concentration force. Instead, another mechanism is at work, opposite to Ricci's (1997). Indeed firms of a given industry located in the country relatively specialized in the other industry are faced with two substitution effects of the same sign, one effect due to the shift of demand from one industry to the other and the other effect to the exchange rate adjustment. The conjunction of these two effects of the same sign turns these firms' production into a convex function of the stochastic parameter representing the industry-specific shock. To have the same average production as firms of the other industry in the same country, these firms can therefore afford to have higher prices and hence higher wages, so that households prefer to work for them: a new dispersion force arises.

Our model thus predicts that because of these conjunctions of substitution and/or income effects, countries tend to be more specialized in a monetary union than under a flexible exchange rate regime. This prediction is not rejected on the empirical side. Some empirical works, such as Fontagné and Freudenberg (1999), reach the conclusion that EMU will not make member countries more specialized simply because previous economic integration has not; but these works fail to acknowledge that the creation of EMU can be modelized as a fall in the transport cost across countries only under Krugman's (1993) assumptions of risk-adverse firms and exogenous exchange rate variations. Other empirical works, such as Frankel and Rose $(1997,1998)$, show that closer international trade linkages have been historically associated with more synchronized national business cycles and conclude that by fostering international trade (as shown by Glick and Rose, 2001; Rose, 2000; Rose and Van Wincoop, 2001), the adoption of a common currency should increase the correlation between national business cycles; but their findings 
are challenged by Imbs (1998) on various grounds, and it remains to be seen whether the kind of international trade fostered by a currency union is intra- or inter-industry trade.

In the remaining of the paper, we first present the model and then examine two particular cases: the concentration of each industry in one country and the dispersion of each industry across countries. A few concluding remarks follow.

\section{The Model}

This section presents our two-country, two-industry monetary model. The two countries considered, labelled home and foreign, share the same structural parameters, including size. The two industries considered produce tradable goods. The choice of only two industries has been made for simplicity: what actually matters is that there are few industries, so that each of them represents a significant share of the productive structure in a country, and thus industry-specific shocks can have non-negligible (income) effects on national economies.

For simplicity, we assume that the endogenous variations of the exchange rate represent the only country-specific-shock-absorber mechanism. This assumption fits EMU quite well, as EMU-members are commonly thought to lack of the adjustment mechanisms which could in theory compensate for the loss of the exchange rate instrument. More precisely, we assume that in the short term, the labour force is immobile between countries (as well as between industries within the same country) and wages are sticky (but prices are flexible). In the long term, the labour force is still immobile between countries, but mobile between industries within the same country, and both wages and prices are flexible. Moreover, our one-period framework rules out the possibility for intertemporal trade, and we also assume that there is no risk-sharing across countries (a good approximation for Europe, as shown for instance by Sørensen and Yosha, 1998): for simplicity we even adopt a "super home bias" assumption, under which every firm redistributes its realized profits equally among its own workers.

Our notations are mainly borrowed from Fujita, Krugman and Venables (1999, ch. 16) and can be shortly described as follows. Superscripts usually refer to households and firms, indexed by integers $i$ and $j$ respectively. Subscripts usually refer to the industry considered ( 1 or 2 ). Variables specific to the foreign country are denoted with an asterix $(*)$. All nominal variables are expressed in local currency. Our presentation focuses on the home country, but equations for the 
foreign country are defined or derived in a similar way.

\section{A. Firms}

We assume monopolistic competition in the goods market, so that each industry is composed of a large number of differentiated tradable goods, called varieties, each firm producing one variety. We note $n_{k}$ and $n_{k}{ }^{*}$ the number of firms in industry $k$ respectively in the home and the foreign country. To keep things simple, we assume that there is the same number $N$ of firms, or equivalently of varieties, in each industry: $N=n_{1}+n_{1} *=n_{2}+n_{2} *$, and that the two countries have a mirrorimage production structure: $n_{1}=n_{2}{ }^{*}$ and $n_{2}=n_{1} *$. This situation is possible since we modelize the two industries in a symmetric way from both the demand and the supply sides (as will be seen below), and since our two countries share the same structural parameters, including size.

We assume the existence of a fixed cost of production, expressed in real terms. As is the rule in the monopolistic competition literature, we modelize this fixed cost as a fixed labour input requirement, noted $F$, the same for both industries and both countries. Each firm produces a variety which is consumed by all households (final consumption) and used by all firms as a production input (intermediate consumption) in both countries. The production functions, which are the same for firms in the same industry but differ between industries, are written

$$
Y_{1}^{j} \equiv A\left(L_{1}^{j}\right)^{\beta}\left(X_{11}^{j}\right)^{\alpha}\left(X_{12}^{j}\right)^{\gamma} \text { and } Y_{2}^{j} \equiv A\left(L_{2}^{j}\right)^{\beta}\left(X_{21}^{j}\right)^{\gamma}\left(X_{22}^{j}\right)^{\alpha}
$$

where, for firm $j$ in industry $k, Y_{k}^{j}$ is the production level, $L_{k}^{j}$ the amount of labour employed (excluding the fixed labour input requirement $F$ ), $X_{k k^{j}}$ the quantity index of intermediate goods bought from industry $k^{\prime}$, and where $\alpha, \beta$ and $\gamma$ are strictly positive parameters such that $\alpha+\beta+\gamma=1$. Following the literature, we impose the condition $\alpha>\gamma$, which says that linkages within industries are stronger than those between industries. We note $\theta$ (with $\theta>1$ ) the constant elasticity of substitution between the different varieties, the same for both industries and for both final and intermediate consumptions, so that $X_{k k^{j}}{ }^{j}$ is defined as

$$
X_{k k^{\prime}}^{j} \equiv\left[\sum_{j^{\prime}=1}^{N}\left(X_{k k^{\prime} k^{\prime}}^{\frac{\theta-1}{\theta}}\right]^{\frac{\theta}{\theta-1}} \text { for }\left(k, k^{\prime}\right) \in\{1,2\}^{2},\right.
$$

where $X_{k k} i j^{\prime \prime}$ represents the amount of goods sold by firm $j$ ' in industry $k$ ' to firm $j$ in industry $k$. 
The timing of events is the following: 1) firms choose their location, 2) wages are set, 3) a shock occurs, 4) monetary policy is implemented, prices are set, production and consumption take place. Step 1 belongs to the long term, while steps 3 and 4 belong to the short term. In other words, wages are assumed to be rigid in the short term, as they do not react to shocks (contrary to prices), and flexible in the long term, as they depend on the pattern of location of firms.

At step 1, that is to say in the long term, firms choose whether to produce their single variety in the home country or in the foreign country. As firms are assumed to behave as risk-neutral agents, each firm chooses the production location which maximizes its expected profit. Now consider a firm in a given industry employing a labour force $l$ (excluding the fixed labour input requirement) paid at wage $w$ : profit-maximization leads to a profit equal to $w l /[\beta(\theta-1)]-w F$, and free entry implies that this profit be nil on average, so that we get:

$$
E\left(L_{k}\right)=F \beta(\theta-1) \text { for } k \in\{1,2\} .
$$

At step 4, as far as price-setting is concerned, following Obstfeld and Rogoff (2000) we rule out local-currency-pricing. In other words, the price of a given good is assumed to be denominated in the producer's currency, as opposed to the buyer's. This ensures that the exchange rate can play its traditional Keynesian expenditure-switching role, because the exchange-rate pass-through to import prices is equal to one. Once the maximization is done, we can drop all indices $j$, as in equilibrium all firms, conditionally to the industry they belong to, end up charging the same price (equal to their marginal cost times a mark-up depending on the elasticity of substitution between goods):

$$
P_{1}=\frac{\theta}{\theta-1}\left(W_{1}\right)^{\beta}\left(G_{1}\right)^{\alpha}\left(G_{2}\right)^{\gamma}, P_{2}=\frac{\theta}{\theta-1}\left(W_{2}\right)^{\beta}\left(G_{1}\right)^{\gamma}\left(G_{2}\right)^{\alpha} \text {, }
$$

with the normalization $A=\left(\alpha^{\alpha} \beta^{\beta} \gamma^{\gamma}\right)^{-1}$, where $W_{k}$ and $G_{k}$ are respectively the nominal wage and the price index in industry $k$ (detailed below).

Finally, we assume that shipping a good from one country to the other is costly. This transport cost, noted $T$, is modelized as an iceberg $\operatorname{cost}(T \geq 1)$. We note $\varepsilon$ the nominal exchange rate, defined as the price of the foreign country currency in terms of the home country currency. The (consumer) price index in industry $k$ is thus written: 


$$
G_{k}=\left[n_{k}\left(P_{k}\right)^{1-\theta}+n_{k}^{*}\left(P_{k}^{*} T \varepsilon\right)^{1-\theta}\right]^{\frac{1}{1-\theta}} \text { for } k \in\{1,2\} .
$$

\section{B. Households}

Each country is composed of a given number $\eta$ of households, who supply labour and consume final goods. The utility function of household $i$ is written

$$
U^{i} \equiv\left(C^{i}\right)^{\lambda}\left(\frac{M^{i}}{P}\right)^{1-\lambda}
$$

where $C^{i}$ is her consumption index, $M^{i}$ her nominal money balance, $P$ the price index, and where $\lambda$ is a constant parameter $(0<\lambda<1)$. Note that for simplicity, total labour $\Lambda^{i} \equiv L^{i}+F^{i}$ supplied by household $i$, where $L^{i}$ represents the productive part and $F^{i}$ the non-productive part (used for the fixed labour input requirement), does not enter the utility function. To compensate for this absence of labour disutility, we impose the average amount of labour supplied by each household $i$ to be exogenously fixed at a given level: $E\left(\Lambda^{i}\right)=\Lambda$. Besides, we assume that labour is demand-determined, workers supplying all the labour demanded by firms following the shock. Each household $i$ works in only one firm, for a wage $W^{i}=W_{k}$ where $k$ is the industry the firm belongs to, so that household $i$ 's budget constraint is thus the following:

$$
M^{i}+P C^{i}=\bar{M}^{i}+P \Psi^{i}+W^{i} \Lambda^{i}+\Pi^{i}
$$

where $\bar{M}^{i}$ and $M^{i}$ denote respectively her initial and final stocks of domestic currency, $\Psi^{i}$ the lump-sum transfer she receives from the central bank (as defined below) and $\Pi^{i}$ the share of her firm's profits she receives under our "super-home bias" assumption. The consumption index of household $i$ is written

$$
C^{i} \equiv\left(C_{1}^{i}\right)^{\mu}\left(C_{2}^{i}\right)^{1-\mu} \text { with } C_{k}^{i} \equiv\left[\sum_{j=1}^{N}\left(C_{k}^{i j}\right)^{\frac{\theta-1}{\theta}}\right]^{\frac{\theta}{1-\theta}} \text { for } k \in\{1,2\},
$$

where $C_{k}{ }^{i}$ is household $i$ 's consumption of goods from industry $k$, and $C_{k}^{i j}$ her consumption of goods of the variety produced by firm $j$ from industry $k$. Parameter $\mu$ is stochastic with mean $E(\mu)=1 / 2$ and variance $V(\mu)$. We assume that its probability distribution is symmetric around $1 / 2$ (so that $\mu$ and $1-\mu$ have the same distribution) and such that $0<\mu<1$ in all cases (so that $V(\mu)<1 / 4$ ). It corresponds to a temporary shock on the relative preference for goods from 
industries 1 and 2, and represents the only source of exogenous perturbations in the model. Finally, the general consumer price index is accordingly $P=\left(G_{l}\right)^{\mu}$ $\left(G_{2}\right)^{1-\mu} /\left[\mu^{\mu}(1-\mu)^{1-\mu}\right]$.

\section{Monetary policy}

We consider two alternative exchange rate regimes: a monetary union and a flexible exchange rate regime. Monetary policy is conducted at the national level in case of a flexible exchange rate regime (one central bank per country) and at the supranational level in case of a monetary union (one central bank for both countries).

The monetary policy transmission mechanism, which says how $P C^{i}$ reacts to $M^{i}$, is described by the first-order condition of the households' optimization problem, that is to say the maximization of (4) with respect to (5):

$$
P C^{i}=\frac{\lambda}{1-\lambda} M^{i}
$$

Moreover, the central bank is assumed to rebate all lump-sum transfers in the form of money: $P \Psi_{i}=M^{i}-\bar{M}^{i}$. Now consider a firm in a given industry employing a labour force $l$ (excluding the fixed labour input requirement) paid at wage $w$ : profit-maximization leads to a profit equal to $w l /[\beta(\theta-1)]-w F$, so that household $i$ 's revenue from working in that firm and receiving a share of its profit is written $W^{i}\left(L^{i}+F^{i}\right)+W^{i} L^{i} /[\beta(\theta-1)]-W^{i} F^{i}=H(1,0) W^{i} L^{i}$, where $H\left(z_{1}, z_{2}\right)$ is defined for each pair of real numbers $z_{1}$ and $z_{2}$ as $H\left(z_{1}, z_{2}\right) \equiv z_{1}[1+\beta(\theta-1)] /[\beta(\theta-1)]$ $+z_{2} / \beta$, and eventually the budget constraint (5) simplifies therefore to:

$$
P C^{i}=H(1,0) W^{i} L^{i}
$$

For simplicity, we do not consider utility-maximizing monetary policies. More precisely, money supply is exogenous (as is standard in the New Open Economy Macroeconomics literature) and kept constant since we ignore monetary policy shocks. Finally, under a flexible exchange rate regime the nominal exchange rate is assumed to adjust so as to balance international trade.

\section{Market clearing}

To derive the goods market clearing conditions (which ensure that international trade is balanced), we first determine total expenditure in the home country on each 
industry. Equation (7) implies that household $i$ spends in nominal terms $H(\mu$, $0) W^{i} L^{i}$ on goods from industry 1 and $H(1-\mu, 0) W^{i} L^{i}$ on goods from industry 2 . Moreover, consider a firm in a given industry employing a labour force $l$ (excluding the fixed labour input requirement) paid at wage $w$ : costs minimization leads this firm to spend $\alpha w l / \beta$ on intermediate goods from the same industry and $z w l / \beta$ on intermediate goods from the other industry. Summing intermediate and final consumptions, we thus obtain the following expressions for $E_{k}$, total expenditure in the home country on industry $k$ :

$$
\begin{gathered}
E_{1}=H(\mu, \alpha) n_{1} W_{1} L_{1}+H(\mu, \gamma) n_{2} W_{2} L_{2} \text { and } \\
E_{2}=H(1-\mu, \gamma) n_{1} W_{1} L_{1}+H(1-\mu, \alpha) n_{2} W_{2} L_{2},
\end{gathered}
$$

where $L_{k}$ is the amount of labour employed (excluding the fixed labour input requirement) by each firm in industry $k$.

Now consider a firm in industry $k$, charging price $p$ and employing a labour force $l$ (excluding the fixed labour input requirement) paid at wage $w$. The nonfixed part of its production costs being $w l / \beta$, the nominal value of its production is $\theta w l /[\beta(\theta-1)]$. Total expenditure on this variety, expressed in the home country currency, is $p^{1-\theta}\left(G_{k}\right)^{\theta-1} E_{k}$ in the home country and $(p T / \varepsilon)^{1-\theta}\left(G_{k}{ }^{*}\right)^{\theta-1} \varepsilon E_{k}{ }^{*}$ in the foreign country. Goods market clearing implies for this variety that total expenditure should be equal to the nominal value of production, so that we eventually obtain:

$$
\frac{\theta}{\theta-1} \frac{W_{k} L_{k}}{\beta}=\left(P_{k}\right)^{1-\theta}\left[G_{k}^{\theta-1} E_{k}+G_{k}^{* \theta-1} T^{1-\theta} \varepsilon^{\theta} E_{k}^{*}\right] \text { for } k \in\{1,2\} .
$$

The presentation of the model is now complete. For simplicity, we focus in the next sections on two particular cases: concentration (defined as each industry entirely located in one country) and dispersion (defined as half of each industry in each country). Note that our framework is thus very close to that of the father of the OCA theory: Mundell (1961) considers first a shift in demand from the goods produced in a country to the goods produced in another (this corresponds to our concentration case), then a shift in demand from goods like lumber products, produced in the Western parts of both Canada and the United States, to goods like cars, produced in the Eastern parts of both countries (our dispersion case). 


\section{The Sustainability of Concentration}

This section focuses on the effect of the exchange rate regime on the sustainability of concentration, or equivalently on the sustainability of endogenously asymmetric demand shocks.

\section{A. General case}

Concentration corresponds to all of industry 1 in the home country and all of industry 2 in the foreign country. Thus $N=n_{1}=n_{2} *$ and $n_{1} *=n_{2}=0$, so that consumer price equations (3) become

$$
G_{1}=N^{\frac{1}{1-\theta}} P_{1}, G_{2}=N^{\frac{1}{1-\theta}} P_{2}^{*} T \varepsilon, G_{1}^{*}=\frac{1}{N^{1-\theta}} P_{1} \frac{T}{\varepsilon}, G_{2}^{*}=\frac{1}{N^{1-\theta}} P_{2}^{*}
$$

Total expenditures (8), in each country and for each industry, are now written

$$
\begin{aligned}
& E_{1}=N W_{1} L_{1} H(\mu, \alpha), E_{2}=N W_{1} L, H(1-\mu, \gamma), \\
& E_{1}^{*}=N W_{2}^{*} L_{2}^{*} H(\mu, \gamma), E_{2}^{*}=N W_{2}^{*} L_{2}^{*} H(1-\mu, \alpha),
\end{aligned}
$$

and using (10), the goods market clearing conditions (9) simplify to $\theta N W_{1} L_{1} /[\beta(\theta-$ 1) $]=E_{1}+\varepsilon E_{1}{ }^{*}$ and $\theta N W_{2}{ }^{*} L_{2}{ }^{*} /[\beta(\theta-1)]=E_{2 / \varepsilon}+E_{2}{ }^{*}$, so that we obtain the following balanced trade condition:

$$
\frac{W_{1} L_{1}}{W_{2}^{*} L_{2}^{*}}=\varepsilon \frac{H(\mu, \gamma)}{H(1-\mu, \gamma)}
$$

In order to examine the sustainability of concentration, we need to consider the case of one marginal firm from industry 2 settling in the home country. Using (2), (10) and (11), the corresponding goods market clearing condition in (9) is written:

$$
\begin{gathered}
\frac{\theta}{(\theta-1)} \frac{W_{2} L_{2}}{\beta}=\left(\frac{W_{2}^{*}}{W_{2}}\right)^{\beta(\theta-1)} T^{-(\theta-1)(\alpha-\gamma)} \varepsilon^{\beta(\theta-1)} \\
{\left[T^{\theta-1} W_{1} L_{1} H(1-\mu, \gamma)+T^{(\theta-1)} \varepsilon W_{2}^{*} L_{2}^{*} H(1-\mu, \alpha)\right],}
\end{gathered}
$$

and using equation (1) we eventually get the following wage ratio equation:

$$
\begin{gathered}
\left(\frac{W_{2}}{W_{1}}\right)^{\beta(\theta-1)+1}=\frac{T^{(\theta-1)(\alpha-\gamma)}}{\theta F}\left(\frac{W_{2}^{*}}{W_{1}}\right)^{\beta(\theta-1)} \\
E\left[\varepsilon^{\beta(\theta-1)}\left[T^{\theta-1} L_{1} H(1-\mu, \gamma)+T^{(\theta-1)} \frac{W_{2}^{*}}{W_{1}} \varepsilon L_{2}^{*} H(1-\mu, \alpha)\right]\right] .
\end{gathered}
$$


Now consider household $i$ in the home country. She can be employed either in one firm from industry 1 or in that marginal firm from industry 2 . She will choose the first alternative if and only if it provides her a higher expected utility level than the second alternative, that is to say, given equation (7), if and only if $W_{2} E\left[L_{2}^{i}\right] \leq$ $W_{1} E\left[L_{l}^{i}\right]$, or equivalently, since $E\left(L_{2}^{i}\right)=E\left(L_{l}{ }^{i}\right)$, if and only if $W_{2} \leq W_{1}$. Equation (14) is therefore central to our analysis: concentration will be sustainable if and only if the right-hand side of this wage ratio equation is lower than one.

\section{B. Cases of Monetary union (mu), Flexible exchange rate refime (fe), and Benchmark Case (bk)}

In a monetary union, the exchange rate is fixed and normalized to one: $\varepsilon=1$. The common central bank sets $M+M^{*}=\bar{M}+\bar{M}^{*}$. In each country, the money stock is linked to consumption through the first-order condition (6) of the households' optimization problem, and consumption in turn is related to income through households' budget constraint (7), so that we get: $W_{1} L_{1}+W_{2} * L_{2} *=\lambda$ $\left(\bar{M}+\bar{M}^{*}\right) /[N(1-\lambda) H(1,0)]$. Together with equations (12) and (1), this leads to $W_{1}=W_{2}{ }^{*}$,

$$
L_{1}=F \beta(\theta-1) \frac{H(\mu, \gamma)}{H\left(\frac{1}{2}, \gamma\right)} \text { and } L_{2}^{*}=F \beta(\theta-1) \frac{H(1-\mu, \gamma)}{H\left(\frac{1}{2}, \gamma\right)}
$$

The wage ratio equation (14) can thus be rewritten in the following way:

$$
\left(\frac{W_{2}}{W_{1}}\right)_{m u}^{\beta(\theta-1)+1}=a_{m u} T^{(\theta-1)(1-\alpha+\gamma)}+b_{m u} T^{(\theta-1)(1+\alpha-\gamma)},
$$

where subscript " $m u$ " refers to "monetary union" and where $a_{m u}$ and $b_{m u}$ are given in appendix A.

Under a flexible exchange rate regime, national central banks remain passive: $M$ $=\bar{M}$ and $M^{*}=\bar{M}^{*}$. For simplicity, we impose $\bar{M}=\bar{M}^{*}$ with no loss of generality. Using (6) and (7), we get $W_{1} L_{1}=\lambda \bar{M} /[N(1-\lambda) H(1,0)]$ and $W_{2} * L_{2} *=\lambda \bar{M} /[N(1-$ ג) $H(1,0)]$. Together with equations (12) and (1), this leads to $W_{1}=W_{2}{ }^{*}$,

$$
L_{1}=L_{2}^{*}=F \beta(\theta-1) \quad \text { and } \quad \varepsilon=\frac{H(1-\mu, \gamma)}{H(\mu, \gamma)} .
$$

The wage ratio equation (14) can thus be rewritten in the following way:

$$
\left(\frac{W_{2}}{W_{1}}\right)_{f e}^{\beta(\theta-1)+1}=a_{f e} T^{(\theta-1)(1-\alpha+\gamma)}+b_{f e} T^{(\theta-1)(1+\alpha-\gamma)},
$$


where subscript " $f e$ " refers to "flexible exchange rate regime" and where $a_{f e}$ and $b_{f e}$ are given in appendix A.

In addition to these two alternative exchange rate regimes, we consider the deterministic case, corresponding to $V(\mu)=0$, as a benchmark case. This benchmark case is very close to Fujita, Krugman and Venables' (1999, ch. 16) framework, the only difference being that they choose to express the fixed cost of production in terms of the composite input (including labour and intermediate goods), whereas we choose to express it in terms of labour only. The wage ratio equation in this benchmark case (subscript " $b k$ ") is obtained by replacing $\mu$ by $1 / 2$ in either wage ratio equation above:

$$
\left(\frac{W_{2}}{W_{1}}\right)_{b k}^{\beta(\theta-1)+1}=a_{b k} T^{(\theta-1)(1-\alpha+\gamma)}+b_{b k} T^{(\theta-1)(1+\alpha-\gamma)},
$$

where $a_{b k}$ and $b_{b k}$ are given in appendix A.

\section{Sustain points}

The values of $T$ strictly higher than one and such that $W_{1}=W_{2}$ are called "sustain points". Appendix B shows from the wage ratio equations (16), (18) and (19) that: i) there exists one unique sustain point $T_{b k}{ }^{S}$ in the $b k$ case, with concentration being sustainable for $T<T_{b k}{ }^{S}$ and unsustainable for $T>T_{b k}{ }^{S 2}$; ii) there exists one unique sustain point $T_{m u}{ }^{S}$ in the $m u$ case, with concentration being sustainable for $T<T_{m u}{ }^{S}$ and unsustainable for $T>T_{m u}{ }^{S}$; iii) there exist two sustain points $T_{f e}{ }^{S}$ and $T_{f e}{ }^{S}$ ' in the $f e$ case, provided that $V(\mu)$ is small enough, with concentration being sustainable for $T_{f e}{ }^{S}<T<T_{f e}{ }^{S}$, and unsustainable for $T<T_{f e}{ }^{S}$ or $T>T_{f e}{ }^{S}$; iv) $T_{f e}{ }^{S}<T_{f e}{ }^{S}{ }^{\prime}<T_{b k}{ }^{S}<T_{m u}{ }^{S}$. These results, which are summarized in figure 1, say that the range of parameter $T$ for which concentration is sustainable is the largest in the $m u$ case and the smallest in the $f e$ case, the benchmark case being intermediate. In other words, a monetary union tends to strengthen the sustainability of concentration, compared to a flexible exchange rate regime.

In the $b k$ case, familiar to the New Economic Geography literature, two concentration forces can be identified, which correspond to the incentive for a firm to settle in the neighbourhood of other firms of the same industry in order to

\footnotetext{
${ }^{2}$ In particular, there is no need for imposing a no-black-hole condition on the parameters, as is done in Fujita, Krugman and Venables (1999), to rule out the possibility for concentration to be sustainable for all $T \mathrm{~s}$ in the $b k$ case. This minor difference between their results and ours is due to different assumptions on the nature of the fixed cost of production.
} 
Figure 1. Sustain points.

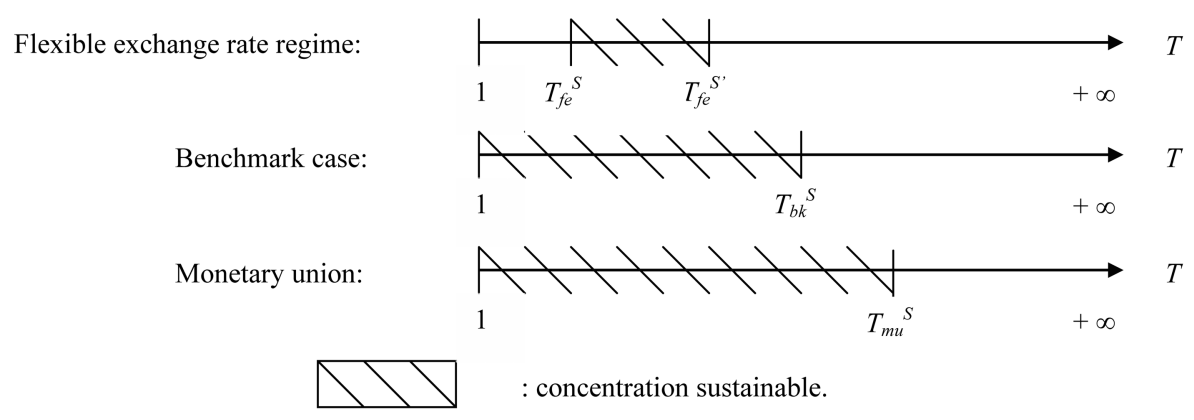

benefit there from cheaper intermediate goods (forward linkage) and from a larger market (backward linkage). Opposite to these concentration forces is a dispersion force which stems from a local competition effect, as firms of a given industry have an incentive to settle in the country not specialized in this industry in order to supply this market at a lower price than their competitors from the other country. The existence of $T_{b k}{ }^{S}$ comes from the fact that the concentration forces are stronger than the dispersion force at low values of $T$, while inversely the dispersion force dominates the concentration forces at high values of $T$.

The $m u$ case adds one concentration force to the $b k$ case. As apparent in equation (15), a monetary union does not smooth asymmetric shocks. As a consequence, when $\mu>1 / 2$ for instance, the marginal firm of industry 2 located in the home country is faced with both a negative substitution effect, since demand shifts from goods of industry 2 to goods of industry 1 , and a positive income effect, since the firm's local market gets larger as the home country specialized in industry 1 gets wealthier (and this matters in the presence of transport costs). The conjunction of these two opposite effects turns this firm's production into a function concave in $\mu$, as apparent in equation (13) if $L_{1}$ and $L_{2}{ }^{*}$ are expressed as in (15). Equation (13) implies therefore that if $W_{2}$ was the same in the $b k$ and $m u$ cases, then this firm's production would be lower on average in the $m u$ case than in the $b k$ case. But free entry and competition between firms ensure that this cannot happen, since in both cases this firm's average production should be equal to the average production of a firm from the other industry in the same country, as stated by (1). Therefore, as apparent in equation (13), $W_{2}$ has to be lower in the $m u$ case than in the $b k$ case for this firm's average production to be identical in both cases. Households in the home country are therefore less attracted into industry 2 in the $m u$ case than in the $b k$ case. In other words, a new concentration force arises in a 
monetary union.

The $f e$ case adds one dispersion force to the $b k$ case. As apparent in equation (17), asymmetric shocks are perfectly smoothed under a flexible exchange rate regime, so that the income effect and consequently the concentration force identified in the $m u$ case withdraw in the $f e$ case. Instead, when $\mu>1 / 2$ for instance, the marginal firm of industry 2 located in the home country is faced with two negative substitution effects, one due to the shift of demand from goods of industry 2 to goods of industry 1 , and the other due to the appreciation of the home country currency, that is to say the decrease in $\varepsilon$ in equation (17). The conjunction of these two effects of the same sign turns this firm's production into a function convex in $\mu$, as apparent in equation (13) if $\varepsilon$ is expressed as in (17). Following the same reasoning as in the $m u$ case, we thus reach the conclusion that $W_{2}$ has to be higher in the $f e$ case than in the $b k$ case. Households in the home country are therefore more attracted into industry 2 in the $f e$ case than in the $b k$ case. In other words, a new dispersion force arises under a flexible exchange rate regime.

These additional concentration force in the $m u$ case and dispersion force in the $f e$ case explain why $T_{f e}{ }^{S},<T_{b k}{ }^{S}<T_{m u}{ }^{S}$. Finally, the non-sustainability of concentration for $T<T_{f e}{ }^{S}$ in the $f e$ case comes from the fact that backward and forward linkages as well as the local competition effect loose their strength for low values of $T$, so that the traditional concentration and dispersion forces are then dominated by our new dispersion force whose existence does not rest on that of transport costs.

\section{Welfare analysis}

Using (6), we get $U=C_{1}{ }^{\mu} C_{2}{ }^{1-\mu}[(1-\lambda) / \lambda]^{1-\lambda}$ where $C_{1}$ and $C_{2}$ are obtained from $G_{l} C_{1}=H(\mu, 0) N W_{1} L_{1}$ and $G_{2} C_{2}=H(1-\mu, 0) N W_{1} L_{1}$, with $G_{1}=N^{l /[-\beta(\theta-1)]}[\theta /(\theta-1)]^{1 /}$ ${ }^{\beta} W_{1} T^{(1-\beta) / \beta} \varepsilon^{(1-\beta) /(1-\alpha+\gamma)}$ and $G_{2}=N^{1 /[-\beta(\theta-1)]}[\theta /(\theta-1)]^{1 / \beta} W_{1} T^{1 / \beta} \varepsilon^{1 /(1-\alpha+\gamma)}$ as implied by equations (2) and (10). Noting that $N=\eta \Lambda /[F[1+\beta(\theta-1)]]$ and using alternatively (15) and (17), we eventually obtain $U$ as a function of the exogenous parameters in the $b k, m u$ and $f e$ cases respectively:

$$
\begin{gathered}
U_{b k}^{c o n c}=\left(\frac{1-\lambda}{\lambda}\right)^{1-\lambda}(\eta \Lambda)^{H(1,0)}[F[1+\beta(\theta-1)]]^{-\frac{1}{\beta(\theta-1)}}\left(\frac{\theta}{\theta-1}\right)^{-\frac{1}{\beta}} \frac{T^{-\left(\frac{1}{2}+\frac{\gamma}{\beta}\right)}}{2} \\
U_{m u}^{c o n c}(\mu)=U_{b k}^{c o n c} 2 \mu^{\mu}(1-\mu)^{1-\mu} T^{\mu-\frac{1}{2}} \frac{H(\mu, \gamma)}{H\left(\frac{1}{2}, \gamma\right)},
\end{gathered}
$$




$$
U_{f e}^{c o n c}(\mu)=U_{b k}^{c o n c} 2 \mu^{\mu}(1-\mu)^{1-\mu} T^{\mu-\frac{1}{2}}\left[\frac{H(\mu, \gamma)}{H(1-\mu, \gamma)}\right]^{\frac{\gamma \mu+(1-\alpha)(1-\mu)}{\gamma+(1-\alpha)}}
$$

Equation (20) shows that compared to the $b k$ case, the $m u$ case is welfareimproving on average when countries are fully specialized (i.e. $E\left[U_{m u}{ }^{c o n c}(\mu)\right]>$ $\left.U_{b k}{ }^{c o n c}\right)$ for two reasons. One reason is that in the presence of industry-specific demand shocks, the differentiated good which matters less in the utility function is less consumed. This consumption basket effect corresponds to the factor $2 \mu^{\mu}(1-$ $\mu)^{(1-\mu)}$, which is larger than one whatever $\mu$ in-between zero and one. The other reason is the conjunction of the substitution effect and the income effect identified in the previous subsection. The substitution effect makes the consumer price index in the home country decrease when $\mu>1 / 2$ (because the differentiated good produced abroad, i.e. the most expensive differentiated good, is then less consumed) and corresponds to the factor $T^{\mu-1 / 2}$ in (20). The income effect makes labour employed in the home country increase when $\mu>1 / 2$, as shown by equation (15), and corresponds to the factor $H(\mu, \gamma) / H(1 / 2, \gamma)$ in (20). The product of these two factors is a strictly convex function of $\mu$, so that Jensen's inequality implies $E\left[T^{\mu-1 / 2} H(\mu, \gamma) / H(1 / 2, \gamma)\right]>1$.

Equation (21) displays the same factors $2 \mu^{\mu}(1-\mu)^{(1-\mu)}$ and $T^{\mu-1 / 2}$ as equation (20), corresponding to the same consumption basket effect and substitution effect respectively. The last factor in equation (21), which corresponds to the contribution of the nominal exchange rate $\varepsilon=H(1-\mu, \gamma) / H(\mu, \gamma)$, depends ambiguously on $\mu$ at first sight because of two opposite effects: indeed, in case of a positive demand shock on industry 1 , households in the home country can afford more foreign goods as their currency appreciates $(\varepsilon<1)$ at the very moment when they are less willing to consume foreign goods. Finally, it is easily shown that $E\left[U_{f e}{ }^{c o n c}(\mu)\right]<$ $E\left[U_{m u}{ }^{c o n c}(\mu)\right]$ or $E\left[U_{f e}{ }^{c o n c}(\mu)\right]>E\left[U_{m u}{ }^{c o n c}(\mu)\right]$ depending on the values of the exogenous parameters.

\section{The Stability of Dispersion}

This section focuses on the effect of the exchange rate regime on the stability of dispersion, or equivalently on the emergence of endogenously asymmetric demand shocks. 


\section{A. General case}

Dispersion corresponds to half of each industry established in each country. We note $n \equiv n_{1}=n_{2} *=n_{1} *=n_{2}=N / 2$. Thus both countries are perfectly identical to each other. This implies that even when flexible, the exchange rate keeps equal to one, whatever the shock on $\mu$ (provided the normalisation $\bar{M}=\bar{M}^{*}$ is done). By symmetry, we have $W_{1}=W_{2}=W_{1}^{*}=W_{2}^{*}$. Together with equations (2) and (3), this implies that producer prices and consumer price indexes are equal across industries and across countries. We note $W \equiv W_{1}=W_{2}=W_{1}^{*}=W_{2}^{*}, P \equiv P_{1}=$ $P_{2}=P_{1}{ }^{*}=P_{2}{ }^{*}$ and $G \equiv G_{1}=G_{2}=G_{1}{ }^{*}=G_{2}{ }^{*}$. Labour employed is derived in the same way as in the previous section, by using equations (1), (6), (7), (9) and noticing that by symmetry $L_{k}=L_{k}{ }^{*}$ for $k \in\{1,2\}$ :

$$
L_{1}=L_{1}^{*}=F \beta(\theta-1) \frac{H(\mu, \gamma)}{H\left(\frac{1}{2}, \gamma\right)} \text { and } L_{2}=L_{2}^{*}=F \beta(\theta-1) \frac{H(1-\mu, \gamma)}{H\left(\frac{1}{2}, \gamma\right)} \text {. }
$$

In order to examine the stability of dispersion, we consider a change in $n_{l}$, noted $d n \neq 0$, leading to a change in $W_{l}$, noted $d W$. By symmetry, we have $d n \equiv d n_{1}=$ $-d n_{2}=-d n_{1} *=d n_{2} *$ and $d W \equiv d W_{1}=-d W_{2}=-d W_{1} *=d W_{2} *$. Dispersion will be stable if and only if $d W / d n \leq 0$.

\section{B. Cases $m u, f e$ and $b k$}

As shown in appendix $\mathbf{C}$, we obtain in the $b k, m u$ and $f e$ cases respectively:

$$
\begin{aligned}
& Q_{b k}(z) \frac{d W}{W}=R_{b k}(Z) \frac{d n}{n}, \\
& Q_{m u}(z) \frac{d W}{W}=R_{m u}(Z) \frac{d n}{n}, \\
& Q_{f e}(z) \frac{d W}{W}=R_{f e}(Z) \frac{d n}{n},
\end{aligned}
$$

where $Z \equiv\left[1-T^{l-\theta}\right] /\left[1+T^{l-\theta}\right]$ and where $Q_{b k}(Z), Q_{m u}(Z), Q_{f e}(Z), R_{b k}(Z), R_{m u}(Z)$, $R_{f e}(Z)$ are functions of $Z$ given in appendix $\mathbf{C}$.

\section{Break points}

The values of $T$ strictly higher than one and such that $d W / d n=0$ are called "break points". Appendix D shows from equations (23), (24) and (25) that: i) there 
Figure 2. Break points.

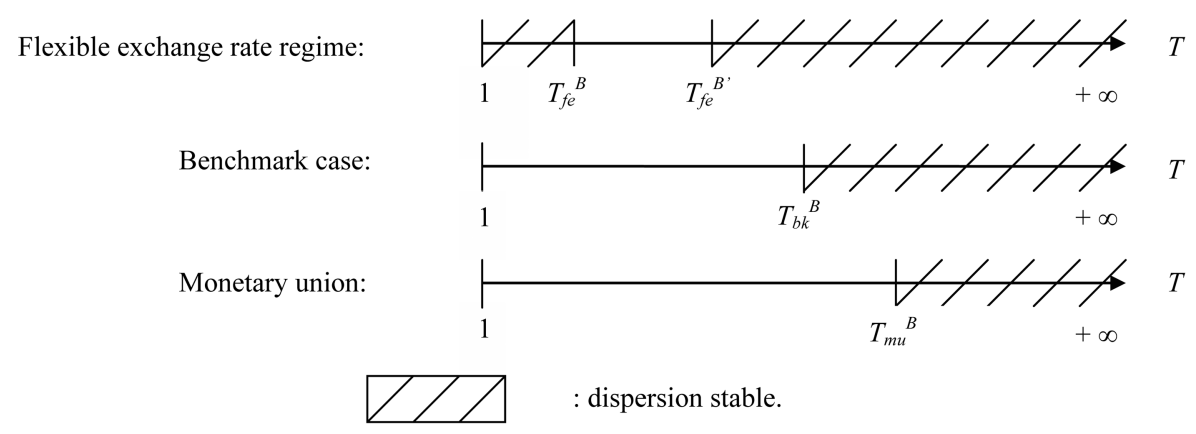

exists one unique break point $T_{b k}{ }^{B}$ in the $b k$ case, with dispersion being stable for $T$ $>T_{b k}{ }^{B}$ and unstable for $T<T_{b k}{ }^{B}$; ii) there exists one unique break point $T_{m u}{ }^{B}$ in the $m u$ case, provided that $V(\mu)$ is small enough, with dispersion being stable for $T>$ $T_{m u}{ }^{B}$ and unstable for $T<T_{m u}{ }^{B}$; iii) there exist two break points $T_{f e}{ }^{B}$ and $T_{f e}{ }^{B}$ ' in the $f e$ case, provided that $V(\mu)$ is small enough, with dispersion being stable for $T<$ $T_{f e}{ }^{B}$ or $T>T_{f e}{ }^{B}$, and unstable for $T_{f e}{ }^{B}<T<T_{f e}{ }^{B}$; iv) $T_{f e}{ }^{B}<T_{f e}{ }^{B}<T_{b k}{ }^{B}<T_{m u}{ }^{B}$. These results, which are summarized in figure 2, say that the range of parameter $T$ for which dispersion is stable is the largest in the $f e$ case and the smallest in the $m u$ case, the $b k$ case being intermediate. In other words, a flexible exchange rate regime tends to strengthen the stability of dispersion, compared to a monetary union.

The mechanism which makes dispersion more unstable in the $m u$ case than in the $b k$ case is the same as the one which makes concentration more sustainable in the $m u$ case than in the $b k$ case: as countries start specializing, industry-specific demand shocks start having a country-specific component which cannot be smoothed by an exchange rate adjustment, and this gives birth to the newly identified concentration force. Similarly, the mechanism which makes dispersion more stable in the $f e$ case than in the $b k$ case is the same as the one which makes concentration less sustainable in the $f e$ case than in the $b k$ case: as countries start specializing, industry-specific demand shocks start having a country-specific component which is smoothed by exchange rate variations, and this gives birth to the newly identified dispersion force. Finally, the stability of dispersion for $T<T_{f e}{ }^{B}$ in the $f e$ case is explained in exactly the same way as the non-sustainability of concentration for $T<T_{f e}^{S}$ in the fe case. 


\section{Welfare analysis}

Using (6), we get $U=C_{1}{ }^{\mu} C_{2}{ }^{1-\mu}[(1-\lambda) / \lambda]^{1-\lambda}$ where $C_{1}$ and $C_{2}$ are obtained from $G C_{1}=n W\left(L_{1}+L_{2}\right) H(\mu, 0)$ and $G C_{2}=n W\left(L_{1}+L_{2}\right) H(1-\mu, 0)$, with $G$ derived from equations (2) and (3). Noting that $N=\eta \Lambda /[F[1+\beta(\theta-1)]]$ and using (22), we eventually obtain $U$ as a function of the exogenous parameters in the $b k, m u$ and $f e$ cases respectively:

$$
\begin{gathered}
U_{b k}^{d i s p}=\left(\frac{1-\lambda}{\lambda}\right)^{1-\lambda}\left(\frac{\eta \Lambda}{2}\right)^{H(1,0)}\left(\frac{\theta}{\theta-1}\right)^{-\frac{1}{\beta}}\left[\frac{1+T^{-1(\theta-1)}}{F[1+\beta(\theta-1)]}\right]^{\frac{1}{\beta(\theta-1)}}, \\
U_{m u}^{d i s p}(\mu)=U_{f e}^{d i s p}(\mu)=U_{b k}^{d i s p} 2 \mu^{\mu}(1-\mu)^{(1-\mu)} .
\end{gathered}
$$

These results simply say that when countries are perfectly diversified, the exchange rate regime does not matter (i.e. $U_{m u}{ }^{\text {disp }}(\mu)=U_{f e}{ }^{d i s p}(\mu)$ for all $\mu$ inbetween zero and one) as countries are affected by industry-specific demand shocks in exactly the same way, and the occurrence of industry-specific demand shocks is always welfare-improving (i.e. $U_{b k}{ }^{d i s p}<U_{m u}{ }^{\text {disp }}(\mu)=U_{f e}{ }^{d i s p}(\mu)$ for all $\mu$ in-between zero and one) due to the same consumption basket effect as in the previous section.

Finally, it is easily shown that $U_{b k}^{\text {conc }}>U_{b k}{ }^{\text {disp }}$ if and only if $2 T^{(1+\alpha-\gamma)(\theta-1) / 2}>$ $1+T^{\theta-1}$, which implies that in the absence of shocks, concentration is more desirable than dispersion for low values of $T$ and less desirable than dispersion for high values of $T$. Naturally, this result holds by continuity in the presence of industry-specific demand shocks, whatever the exchange rate regime, provided that $V(\mu)$ is small enough. Now as shown in figures 1 and 2, for low values of $T$ and $V(\mu)$ a monetary union makes concentration sustainable and dispersion unstable while on the contrary a flexible exchange rate regime makes concentration nonsustainable and dispersion stable. This suggests that a monetary union is preferable to a flexible exchange rate regime when $T$ and $V(\mu)$ are small enough.

\section{Conclusion}

In his classic contribution, Mundell (1961) examines in a two-country twoindustry framework how exchange rate regimes should ideally be chosen, given countries' specialization patterns, in the presence of industry-specific demand shocks. In this paper, we inversely consider how exchange rate regimes influence countries' specialization patterns in the presence of industry-specific demand 
shocks. To that aim, we introduce these shocks, together with short-run nominal wage rigidity, into an otherwise canonical New Economic Geography model, that of Fujita, Krugman and Venables (1999, ch. 16).

Our results suggest that the occurrence of industry-specific demand shocks ${ }^{3}$ makes the dispersion of each industry across countries less stable, and the concentration of each industry in one country more sustainable, in a monetary union than under a flexible exchange rate regime. This is because the conjunction of a substitution effect and an income effect gives rise to a concentration force in a monetary union, while the conjunction of two substitution effects gives rise to a dispersion force under a flexible exchange rate regime. Thus, endogenously asymmetric demand shocks are more likely to emerge and be sustained in a monetary union than under a flexible exchange rate regime. This result, based on risk-neutral firms and endogenous exchange rate variations, goes in the same direction as Krugman's (1993) prediction, based on risk-adverse firms and exogenous exchange rate variations, and opposite to Ricci's (1997) result, based on risk-adverse firms and endogenous exchange rate variations. We show moreover that such an outcome need not be a cause for concern, as endogenously asymmetric demand shocks in a monetary union are not necessarily welfare-reducing.

Though centered on EMU, our point can naturally be applied to the (still distant) prospect of an East Asian monetary union, all the more so than East Asian countries are less diversified than European countries. Moreover, our analysis has also some implications for regions within a country, as these regions form a currency union, and the smaller the size of the redistributive budget at the national level, the larger the income effect mentioned above, the stronger the newly identified concentration force and therefore the more likely the emergence and sustainability of endogenously asymmetric demand shocks within the country.

\section{Acknowledgements}

I am grateful to Agnès Bénassy-Quéré, Daniel Cohen, Jordi Galí, Philippe Martin and Charles Wyplosz for useful suggestions. The usual disclaimer applies.

Received 9 July 2004, Accepted 26 December, 2004

\footnotetext{
${ }^{3}$ Note that the occurrence of industry-specific productivity shocks would have no impact on the location incentives of firms in our framework. Indeed, prices would adjust to a productivity shock so as to leave labour employed unchanged, thus killing the income effect in the $m u$ case and the substitution effect in the fe case.
} 


\section{References}

Fontagné, L., Freudenberg, M. (1999) Endogenous Symmetry of Shocks in a Monetary Union, Open Economies Review, 10, 263-287.

Frankel, J. A., Rose, A. K. (1997) Is EMU More Justifiable Ex Post than Ex Ante?, European Economic Review, 41, 753-760.

Frankel, J. A., Rose, A. K. (1998) The Endogeneity of the Optimum Currency Area Criteria, The Economic Journal, 108, 1009-1025.

Fujita, M., Krugman, P., Venables, A. J. (1999) The Spatial Economy: Cities, Regions and International Trade, MIT Press, Cambridge.

Glick, R., Rose, A. K. (2001) Does a Currency Union Affect Trade? The Time Series Evidence, European Economic Review, 46, 1125-1151.

Imbs, J. (1999) Fluctuations, Bilateral Trade and the Exchange Rate Regime, Cahiers de recherches économiques du DEEP No. 9906.

Kenen, P. (1969) The Theory of Optimum Currency Areas: an Eclectic View, in Monetary Problems in the International Economy (Ed.) Mundell, R., Swoboda, A., University of Chicago Press, Chicago, pp. 41-60.

Krugman, P. (1993) Lessons from Massachusetts for EMU, in Adjustment and Growth in the European Monetary Union (Ed.) Torres, F., Giavazzi, F., Cambridge University Press, Cambridge, pp. 241-260.

Loisel, O. (2003) Four Essays on Macroeconomic Volatility and Instability under Alternative Exchange Rate Regimes, $\mathrm{PhD}$ Dissertation of the École Polytechnique, Palaiseau.

Mundell, R. A. (1961) A Theory of Optimum Currency Areas, American Economic Review, 51, 657-665.

Obstfeld, M., Rogoff, K. (2000) New Directions for Stochastic Open Economy Models, Journal of International Economics, 50, 117-153.

Ricci, L. A. (1997) Exchange Rate Regimes and Location, IMF Working Paper No. 97/69.

Ricci, L. A. (1998) Uncertainty, Flexible Exchange Rates, and Agglomeration, IMF Working Paper No. 98/9.

Rose, A. K. (2000) One Money, One Market: Estimating the Effect of Common Currencies on Trade, Economic Policy, 30, 7-46.

Rose, A. K., Van Wincoop, E. (2001) National Money as a Barrier to International Trade: the Real Case for Currency Union, American Economic Review, 91, 386-390.

Sørensen, B. E., Yosha, O. (1998) International Risk Sharing and European Monetary Unification, Journal of International Economics, 45, 211-238. 


\section{Appendix}

\section{A. Values of $a_{m u}, b_{m u}, a_{f e}, b_{f e}, a_{b k}$ and $b_{b k}$}

$$
\begin{gathered}
a_{m u} \equiv \frac{\beta(\theta-1)}{\theta} \frac{\left[H\left(\frac{1}{2}, \gamma\right)\right]^{2}-[H(1,0)]^{2} V(\mu)}{H\left(\frac{1}{2}, \gamma\right)}, \\
b_{m u} \equiv \frac{\beta(\theta-1)}{\theta} \frac{H\left(\frac{1}{2}, \alpha\right) H\left(\frac{1}{2}, \gamma\right)+[H(1,0)]^{2} V(\mu)}{H\left(\frac{1}{2}, \gamma\right)}, \\
a_{f e} \equiv \frac{\beta(\theta-1)}{\theta} E\left[\left(\frac{H(1-\mu, \gamma)}{H(\mu, \gamma)}\right)^{\beta(\theta-1)+1} H(\mu, \gamma)\right], \\
b_{f e} \equiv \frac{\beta(\theta-1)}{\theta} E\left[\left(\frac{H(1-\mu, \gamma)}{H(\mu, \gamma)}\right)^{\beta(\theta-1)+1} H(1-\mu, \alpha)\right], \\
a_{b k} \equiv \frac{\beta(\theta-1)}{\theta} H\left(\frac{1}{2}, \gamma\right) \text { and } b_{b k} \equiv \frac{\beta(\theta-1)}{\theta} H\left(\frac{1}{2}, \alpha\right) .
\end{gathered}
$$

\section{B. Sustain points $T_{f e}{ }^{S}, T_{f e}{ }^{S}, T_{b k}{ }^{S}$ and $T_{m u}{ }^{S}$}

For $i \in\{b k, f e, m u\}$, let us note

$$
K_{i}(T) \equiv\left(\frac{W_{2}}{W_{1}}\right)^{\beta(\theta-1)+1}=a_{i} T^{(\theta-1)(1-\alpha+\gamma)}+b_{i} T^{(1+\alpha-\gamma)} .
$$

For $i \in\{b k, m u\}$, we have $K_{i}(1)=1, \lim _{T \rightarrow+\infty} K_{i}(T)=+\infty, \partial K_{i} / \partial T<0$ for $T$ $<b_{i}(1+\alpha-\gamma) /\left[a_{i}(1-\alpha+\gamma)\right]$ and $\partial K_{i} / \partial T>0$ for $T>b_{i}(1+\alpha-\gamma) /\left[a_{i}(1-\alpha+\gamma)\right]$, with $b_{i}(1+\alpha-\gamma) /\left[a_{i}(1-\alpha+\gamma)\right]>1$ since $\alpha>\gamma$ and $b_{i}>a_{i}$, from which we derive the existence of a sustain point $T_{i}^{S}$ such that concentration is sustainable for $T<T_{i}^{S}$ and unsustainable for $T>T_{i}^{S}$.

In the $f e$ case, we have $K_{f e}(1)>1, \lim _{T \rightarrow+\infty} K_{f e}(T)=+\infty, \partial K_{f e} / \partial T<0$ for $T<$ $b_{f e}(1+\alpha-\gamma) /\left[a_{f e}(1-\alpha+\gamma)\right]$ and $\partial K_{f e} / \partial T>0$ for $T>b_{f e}(1+\alpha-\gamma) /\left[a_{f e}(1-\alpha+\gamma)\right]$, with $b_{f e}(1+\alpha-\gamma) /\left[a_{f e}(1-\alpha+\gamma)\right]>1$ since $\alpha>\gamma$ and $b_{f e}>a_{f e}$ (due to Jensen's inequality). Moreover, $\lim _{V(\mu)} \rightarrow{ }_{0} K_{f e}(T)=K_{b k}(T)$ for all $T \geq 1$, so that by continuity we get, provided that $V(\mu)$ is small enough, the existence of two sustain points $T_{f e}{ }^{S}$ and $T_{f e}{ }^{S}$, such that concentration is sustainable for $T_{f e}{ }^{S}<T<T_{f e}{ }^{S}$, and unsustainable for $T<$ $T_{f e}^{S}$ or $T>T_{f e}^{S}$. 
Finally, we find that for all $T>1, K_{m u}(T)<K_{b k}(T)$ and $K_{b k}(T)<K_{f e}(T)$, the latter inequality due to Jensen's inequality (which ensures that $a_{b k}<a_{f e}$ and $b_{b k}<b_{f e}$ ), so that we get $T_{f e}{ }^{S}<T_{f e}{ }^{S}$ ' $<T_{b k}{ }^{S}<T_{m u}$.

\section{Determination of $Q_{b k}(Z), R_{b k}(Z), Q_{m u}(Z), R_{m u}(Z), Q_{f e}(Z)$ and $R_{f e}(Z)$}

In the benchmark case, we have by symmetry $d P_{1} / P=-d P_{2} / P=-d P_{1} * / P=$ $d P_{2} * / P$ and $d G_{1} / G=-d G_{2} / G=-d G_{1} * / G=d G_{2} * / G$. We note $d P / P \equiv d P_{1} / P$ and $d G / G \equiv d G_{I} / G$. Using the producer price equations (2) and the consumer price index equations (3), we easily get $d P / P$ and $d G / G$ as functions of $d n / n$ and $d W / W$ only ( since $d \varepsilon / \varepsilon=0$ ). We then differentiate one of the goods market clearing conditions (9), noticing that $d E_{k}=d E_{k}{ }^{*}=0$ for $k \in\{1,2\}$, because $\mu=1 / 2$ and because $d L_{k}=d L_{k}{ }^{*}=0$ for $k \in\{1,2\}$ due to (1). This differentiated goods market clearing condition corresponds to a relationship between $d P / P, d G / G$ and $d W / W$, so that using the expressions of $d P / P$ and $d G / G$ previously obtained (as functions of $d n / n$ and $d W / W$ ) we finally get equation (23) with

$$
\begin{gathered}
Q_{b k}(Z) \equiv 1+\beta(\theta-1)-(\alpha-\gamma)\left(\frac{2 \theta-1}{\theta}\right) Z+(\theta-1)\left[\frac{(\alpha-\gamma)^{2}}{\theta}-\beta\right] Z^{2} \\
\text { and } R_{b k}(Z) \equiv(\alpha-\gamma)\left(\frac{2 \theta-1}{\theta}\right) Z-\left[1+(\alpha-\gamma)^{2}\left(\frac{\theta-1}{\theta}\right)\right] Z^{2}
\end{gathered}
$$

For a monetary union, since $d \varepsilon / \varepsilon=0$ we get $d P / P \equiv d P_{1} / P=-d P_{2} / P=-d P_{1} * / P$ $=d P_{2} * / P$ and $d G / G \equiv d G_{1} / G=-d G_{2} / G=-d G_{1} * / G=d G_{2} * / G$ as functions of $d n /$ $n$ and $d W / W$ exactly in the same way as in the benchmark case. We then differentiate one of the goods market clearing conditions (9). This time however, $d E_{k} \neq 0$ and $d E_{k}^{*} \neq 0$ for $k \in\{1,2\}$, because $d L_{1}+d L_{2}=-\left(d L_{1}{ }^{*}+d L_{2}{ }^{*}\right)=k(2 \mu-$ 1), where $k$ depends on $d n / n, d W / W, \alpha, \gamma, \theta$ and $T$, as can be shown with the help of the other goods market clearing conditions. We finally obtain equation (24) with

$$
\begin{gathered}
Q_{m u}(Z) \equiv Q_{b k}(Z)+2 \beta^{2} \frac{(\theta-1)^{2}}{\theta} Z(1+Z) \frac{[H(1,0)]^{2}}{H\left(\frac{1}{2}, \gamma\right)} V(\mu) \\
\text { and } R_{m u}(Z) \equiv R_{b k}(Z)+2 \beta \frac{(\theta-1)}{\theta} Z(1+Z) \frac{[H(1,0)]^{2}}{H\left(\frac{1}{2}, \gamma\right)} V(\mu)
\end{gathered}
$$

For a flexible exchange rate regime, computations are simplified by the normalisation $\bar{M}=\bar{M}^{*}$. We first use the producer price equations (2) and the 
consumer price index equations (3) to get $d P_{1} / P, d P_{2} / P, d P_{1} * / P, d P_{2} * / P, d G_{1} / G$, $d G_{2} / G, d G_{1}{ }^{*} / G$ and $d G_{2}{ }^{*} / G$ as functions of $d n / n, d W / W$ and $d \varepsilon / \varepsilon$. We then differentiate the goods market clearing conditions to get $d \varepsilon / \varepsilon=k^{\prime}(1-2 \mu)$, where $k^{\prime}$ depends on $d n / n, d W / W, \alpha, \gamma, \theta$ and $T$. Replacing $d \varepsilon / \varepsilon$ by $k^{\prime}(1-2 \mu)$ in one of the goods market clearing conditions, we finally obtain equation (25) with

$$
\begin{gathered}
Q_{f e}(Z) \equiv Q_{b k}(Z)-\beta(\theta-1)\left(1-Z^{2}\right)\left[\frac{H(1,0)}{H\left(\frac{1}{2}, \gamma\right)}\right]^{2} V(\mu) \\
\text { and } R_{f e}(Z) \equiv R_{b k}(Z)-\left(1-Z^{2}\right)\left[\frac{H(1,0)}{H\left(\frac{1}{2}, \gamma\right)}\right]^{2} V(\mu) .
\end{gathered}
$$

\section{Break points $T_{f e}^{B}, T_{f e}^{B}, T_{b k}^{B}, T_{m u}{ }^{B}$}

We first check that $Q_{b k}(Z)>0, Q_{m u}(Z)>0$ and $Q_{f e}(Z)>0$ for all $T>1$ or equivalently for all $Z \in[0,1]$, since $V(\mu)<1 / 4$. The break points correspond therefore to the values of $T>1$, or equivalently the values of $Z \in[0,1]$, such that $R_{b k}(Z)=0, R_{m u}(Z)=0$ or $R_{f e}(Z)=0$ depending on the case considered. Since $R_{b k}(Z), R_{m u}(Z)$ and $R_{f e}(Z)$ are second-order polynomials in $Z$ and $Z$ is a strictly monotonous function of $T$, these break points are easily determined. We thus find that there exists one unique break point $T_{b k}{ }^{B}$ in the $b k$ case, with dispersion being stable for $T>T_{b k}{ }^{B}$ and unstable for $T<T_{b k}{ }^{B}$; there exists one unique break point $T_{m u}{ }^{B}$ in the $m u$ case, provided that

$$
V(\mu)<V_{m u} \equiv \frac{[1-(\alpha-\gamma)]\left[1-(\alpha-\gamma)\left(\frac{\theta-1}{\theta}\right)\right]}{4 \beta\left(\frac{\theta-1}{\theta}\right) \frac{[H(1,0)]^{2}}{H\left(\frac{1}{2}, \gamma\right)}},
$$

with dispersion being stable for $T>T_{m u}{ }^{B}$ and unstable for $T<T_{m u}{ }^{B}$; and there exist two break points $T_{f e}{ }^{B}$ and $\mathrm{T}_{f e}{ }^{B}$, in the $f e$ case, provided that

$$
V(\mu)<V_{f e} \equiv \frac{1}{2}\left[\frac{H\left(\frac{1}{2}, \gamma\right)}{H(1,0)}\right]^{2}
$$




$$
\left[1+(\alpha-\gamma)^{2}\left(\frac{\theta-1}{\theta}\right)-\sqrt{\left[1-(\alpha-\gamma)^{2}\right]\left[1-(\alpha-\gamma)^{2}\left(\frac{\theta-1}{\theta}\right)^{2}\right]}\right],
$$

with dispersion being stable for $T<T_{f e}^{B}$ or $T>T_{f e}^{B}$, and unstable for $T_{f e}{ }^{B}<T<$ $T_{f e}{ }^{B}$. Though figure 2 focuses on the case $V(\mu)<\operatorname{Min}\left(V_{m u}, V_{f e}, 1 / 4\right)$, it is worth noting that dispersion becomes unstable whatever the value of $T$ in a monetary union when $V(\mu)$ reaches the threshold $V_{m u}$, and that dispersion becomes stable whatever the value of $T$ under a flexible exchange rate regime when $V(\mu)$ reaches the threshold $V_{f e}$.

Finally, we easily show that $T_{f e}^{B}<T_{f e}^{B}{ }^{B}<T_{b k}^{B}<T_{m u}{ }^{B}$, since $R_{f e}(Z)<R_{b k}(Z)<$ $R_{m u}(Z)$ for all $Z \in[0,1]$. Note that contrary to the sustain points, the break points can be explicitly expressed as analytical functions of the structural parameters. 